\title{
New multiresponsive chromic soft materials: dynamic interconversion of short 2,7-dicyanomethylenecarbazole- based biradicaloid and the corresponding cyclophane tetramer
}

Article

Accepted Version

Wang, D., Capel Ferrón, C., Li, J., Gámez-Valenzuela, S., Ponce Ortiz, R., López Navarrete, J. T., Hernández Jolín, V., Yang, X., Peña Álvarez, M., García Baonza, V., Hartl, F., Ruiz Delgado, M. C. and Li, H. (2017) New multiresponsive chromic soft materials: dynamic interconversion of short 2,7dicyanomethylenecarbazole-based biradicaloid and the corresponding cyclophane tetramer. Chemistry- A European Journal, 23 (55). pp. 13776-13783. ISSN 1521-3765 doi: https://doi.org/10.1002/chem.201702659 Available at https://centaur.reading.ac.uk/72388/

It is advisable to refer to the publisher's version if you intend to cite from the work. See Guidance on citing.

To link to this article DOI: http://dx.doi.org/10.1002/chem.201702659

Publisher: Wiley 
All outputs in CentAUR are protected by Intellectual Property Rights law, including copyright law. Copyright and IPR is retained by the creators or other copyright holders. Terms and conditions for use of this material are defined in the End User Agreement.

\section{www.reading.ac.uk/centaur}

\section{CentAUR}

Central Archive at the University of Reading

Reading's research outputs online 
WILEY-VCH

1

2

3

4

5

6

7

8

\title{
New Multi-responsive Chromic Soft Materials: Dynamic Interconversion of Short 2,7-Dicyanomethylenecarbazole-based Biradicaloid and Corresponding Cyclophane Tetramer
}

\author{
Deliang Wang, ${ }^{\mathrm{a}}$ Cristina Capel Ferrón, ${ }^{\mathrm{b}} \mathrm{Jie} \mathrm{Li}$, ${ }^{\mathrm{a}}$ Sergio Gámez-Valenzuela, ${ }^{\mathrm{b}}$ Rocio Ponce Ortiz, ${ }^{\mathrm{b}} \mathrm{Juan} \mathrm{T}$. \\ López Navarrete, ${ }^{\mathrm{b}}$ Víctor Hernández Jolín, ${ }^{\mathrm{b}}$ Xiaodi Yang, ${ }^{\mathrm{c}}$ Miriam Peña Álvarez, ${ }^{\mathrm{d}}$ Valentín García \\ Baonza, ${ }^{d}$ František Hartl, ${ }^{*, e}$ M. Carmen Ruiz Delgado, ${ }^{*, b}$ Hongxiang Li $i^{*, a}$
}

\begin{abstract}
Herein, we report a quinodimethane-type molecule, 2,7dicyanomethylene-9-(2-ethylhexyl)carbazole (1), one of the shortest $\pi$-conjugated biradicaloids reported to be stable in solution at ambient. This carbazole-based quinoidal precursor is able to form a macrocyclic $\sigma$-bonded tetramer (2). The resolved single crystal X-ray structure of tetramer 2 shows that four molecules of 1 are linked together through four long $(\mathrm{CN})_{2} \mathrm{C}-\mathrm{C}(\mathrm{CN})_{2}$ bonds $(1.631 \AA)$ resulting from coupling of the unpaired electrons in biradicaloid 1. Dynamic interconversion between monomer $\mathbf{1}$ and cyclophane tetramer $\mathbf{2}$ is achieved by reversible cleavage and recovery of the four $(\mathrm{CN})_{2} \mathrm{C}-$ $\mathrm{C}(\mathrm{CN})_{2}$ bonds upon soft external stimuli (light absorption, temperature and pressure), which is accompanied by significant colour changes. These novel photo- thermo- and mechano-chromic properties expand the versatility of $\pi$-conjugated biradicaloid compounds as novel functional materials that, in combination with spin chemistry and dynamic covalent chemistry, can be relevant in molecular machines, sensors, and switches.
\end{abstract}

\section{Introduction}

$\pi$-Conjugated biradical compounds, featuring unique unsaturated valences and radical centers in the ground state, are fundamentally important for understanding the nature of chemical bonds and the fundamental physical and chemical properties of $\pi$-conjugated molecules, and have potential applications in material science. ${ }^{[1-3]}$ However, most of the $\pi$-conjugated biradical compounds exhibit high reactivity, easily decomposing or polymerizing under ambient experimental conditions, which preclude the investigation of their properties and applications.

a. Key Laboratory of Synthetic and Self-assembly Chemistry for Organic Functional Materials, Shanghai Institute of Organic Chemistry, Chinese Academy of Sciences, No. 345 Lingling Rd., Shanghai, 200032, China. Address here.

Department of Physical Chemistry, University of Malaga, Campus de Teatinos s/n, 229071, Malaga, Spain.

Laboratory of Advanced Materials, Fudan University, No. 200 Handan Rd., Shanghai, 200438, China.

MALTA-Consolider Team, Department of Physical Chemistry, Complutense University of Madrid, 28040 Madrid (Spain)

e. Department of Chemistry, University of Reading, Whiteknights, Reading RG6 6AD, United Kingdom.

Electronic Supplementary Information (ESI) available. See DOI: XXXXXXXXXXXXXX
Recently, significant progress has been achieved in this field, as some stable $\pi$-conjugated biradicals were prepared through increasing the conjugation length and/or introducing bulky substituents at the radical centers. ${ }^{[4-11]}$

Nonetheless, $\pi$-conjugated biradicals existing at ambient conditions are still scarce. Therefore, rational design and facile syntheses of novel stable biradical, or significantly spin-spin interacting biradicaloid compounds to explore their bonding situation, physical properties, and pertinent applicability, constitute a longstanding prominent challenge. Note that the stability of the biradicaloid compounds depends on which of the resonant structures, the open-shell biradical or the closed-shell quinoidal form, contributes more to the ground state structure.

Dynamic covalent chemistry (DCC) has attracted great attention because it provides an efficient strategy to prepare complex assemblies. [12-13] The building block that can dynamically form covalent bonds is the key for the success of DCC. $\pi$-Conjugated mono- and biradicals are potential building blocks in DDC since they can selectively construct macrocyclic or staircase oligomers or polymers by self-assembly processes. For instance, triphenylamine and carbazole units substituted with dicyanomethyl radicals have been reported to form macrocyclic $\sigma$-bonded oligomers. ${ }^{[14]}$ Another interesting example is the reversible formation of $\sigma$-aggregates observed in quinoidal naphthodithiophenes substituted with cyanoalkyloxycarbonylmethylene groups. ${ }^{[15]}$

Due to their low-lying LUMO, and subsequent small HOMOLUMO energy gaps, quinoidal compounds have found wide applications as organic functional materials. [16-19] With the increasing conjugation length, they exhibit a Kekulé or Tschitschibabin type biradical character in their ground state. ${ }^{[8,20]}$ The shortest stable biradicals reported to date have been fused five-ring quinones or oligo-quinones with four aromatic rings (Supporting Information, Scheme S1).

In this study, we report an unusual fused three-ring biradicaloid 1 (Scheme 1) that is readily prepared from commercially available 2,7-dibromocarbazole. To the best of our knowledge, $\mathbf{1}$ is the smallest stable quinoidal biradicaloid reported to date. Thus, the insertion of terminal dicyanomethylene groups results in a very efficient strategy to stabilize the highly reactive biradical specie while at the same time a quinoidal structure is preserved in the ground state.

Interestingly, biradicaloid 1 reversibly converts upon external stimuli (temperature variation, pressure, light absorption) to cyclophane tetramer $\mathbf{2}$, as a result of the formation (or bond 
cleavage) of highly strained and long $(\mathrm{CN})_{2} \mathrm{C}-\mathrm{C}(\mathrm{CN})_{2}$ bonds, as determined by single-crystal $\mathrm{X}$-ray analysis. This cyclophane tetramer represents the first example of a $\sigma$-aggregated compound formed from a stable quinoidal precursor, featuring

positive: $m / z$ 406.2024). Figure $1 \mathrm{a}-\mathrm{b}$ illustrates the ${ }^{1} \mathrm{H}$ NMR spectra of precursor 3 and quinoidal system 1 prepared by in situ oxidation in $\mathrm{CD}_{2} \mathrm{Cl}_{2}$ a)<smiles>CCC(C)Cn1c2cc(Br)ccc2c2ccc(Br)cc21</smiles>

1

1

b)

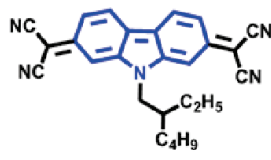

1-CS
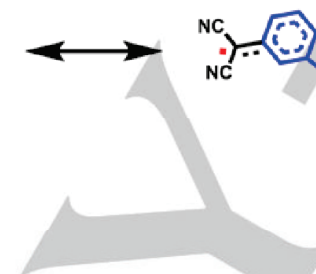

Scheme 1. a) The synthetic route to biradicaloid 1 and corresponding cyclophane-type tetramer 2 ; b) resonance structures of compound 1: the closed-shell singlet state (1-CS) and the open-shell singlet biradical state (1-OS).

photo-, thermo-, and mechano-chromic properties. The dynamic and reversible interconversion between biradicaloid 1 and cyclophane tetramer $\mathbf{2}$ is revealed here by combined UV-Vis, IR, Raman, EPR and ${ }^{1} \mathrm{HNMR}$ spectral data, with support from DFT calculations.

\section{Results and Discussion}

\section{Syntheses and Structural Characterization}

The synthetic route to quinoidal compound 1 and cyclophane tetramer $\mathbf{2}$ is shown in Scheme 1. 2,7-dibromo-9-(2-ethylhexyl) carbazole $(\mathrm{DBC})$ reacted with malononitrile through a typical $\mathrm{Pd}-$ catalyzed Takahashi coupling reaction to afford compound 3 as a faint yellow solid. When compound 3 was oxidized with 2,3dichloro-5,6-dicyanobenzoquinone (DDQ), a deep violet solution was obtained. The violet reaction mixture was quickly chromatographed on a silica gel column and a purple solution of monomer 1 in dichloromethane was collected. When the solvent (dichloromethane) was removed under vacuum, compound 1 fully converted to cyclophane tetramer $\mathbf{2}$ obtained as a white powder.

When tetramer 2 was re-dissolved in dichloromethane, it partially converted back to $\mathbf{1}$, as evidenced by the recovery of the purple color.

The chemical structure of quinoidal compound 1 has been characterized with ${ }^{1} \mathrm{H}$ NMR (Figure 1) and high resolution mass spectrometry (Figure S1, Supporting Information- DART
Both compounds showed conspicuous proton signals. Compared to compound $\mathbf{3}$, the protons in $\mathbf{1}$ in the range of $7.0-8.5 \mathrm{ppm}$ are shifted to the lower field region, and the signal at $5.30 \mathrm{ppm}$, which is ascribed to the proton of the dicyanomethyl group in compound 3, has disappeared; in addition, the proton signals of $\mathbf{1}$ have broadened. This broadening is ascribed to a thermally accessible triplet biradical state (see below). The time-progression of the ${ }^{1} \mathrm{H}$ NMR spectra indicated that compound 1 oligomerized gradually in $\mathrm{CD}_{2} \mathrm{Cl}_{2}$ in the presence of $\mathrm{DDQ}$ (Supporting Information, Figures S2-S3); the oligomerization accelerated upon increase of the solution temperature. In contrast, the UV-Vis absorption spectra showed that pure compound 1 was stable at ambient conditions in diluted $\mathrm{CHCl}_{3}$ solution for several months, as discussed below. Single crystals of compound 2 were prepared through solvent evaporation from a solution of compound 1 (Figure 2, and Figure S4).

The single crystal X-ray analysis has revealed that in $\mathbf{2}$ four molecules of parent compound $\mathbf{1}$ are linked together through four unusually long $(\mathrm{CN})_{2} \mathrm{C}-\mathrm{C}(\mathrm{CN})_{2}$ bonds $(1.631 \AA)$ by pairwise coupling of the eight unpaired electrons, thereby creating a cavity structure of $12.52 \AA$ length. This behaviour provides an evidence of the biradicaloid nature of $\mathbf{1}$ in the ground state. The cavity parameters of tetramer $\mathbf{2}$ indicate its capacity for inclusion of large molecules (e.g., $\mathrm{C}_{60}$ or $\mathrm{C}_{70}$ ) and, therefore, its potential applications in the area of molecular machines, sensors, and switches. ${ }^{[21-22]}$ The $(\mathrm{CN})_{2} \mathrm{C}-\mathrm{C}(\mathrm{CN})_{2}$ bonds between the monomer 
components of tetramer $\mathbf{2}$ are significantly longer than prototypical $\mathrm{C}\left(\mathrm{sp}^{3}\right)-\mathrm{C}\left(\mathrm{sp}^{3}\right)$ bonds $(1.54 \AA)$, suggesting their relative weakness and hence the possibility of bond cleavage in response to external stimuli, as will be demonstrated in the following sections.
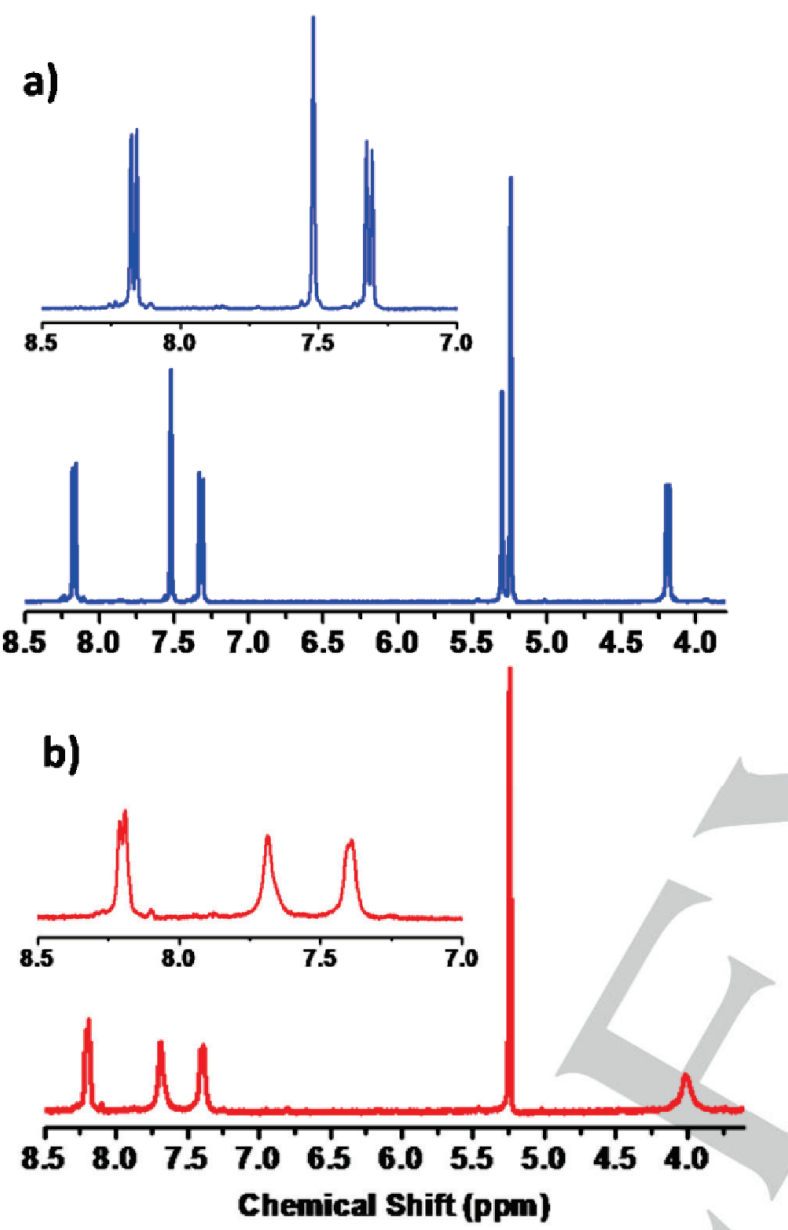

Figure 1. The ${ }^{1} \mathrm{H}$ NMR spectra of compound 3 (a) and quinoidal compound 1 (b) in $\mathrm{CD}_{2} \mathrm{Cl}_{2}$. 1 was prepared in situ by addition of DDQ to compound $\mathbf{3}$ in the NMR tube.

The DFT-calculated bond lengths of tetramer $\mathbf{2}$ are in good accordance with the X-ray diffraction data (Supporting Information, Figure S5). The aromaticity of the carbazole unit in tetramer $\mathbf{2}$ is fully recovered and the $\mathrm{CN}$ groups are linked to $\mathrm{sp}^{3}$ carbons, indicating that the transformation of biradicaloid monomers 1 to tetramer 2 involves two processes: (i) a quinoidal-to-aromatic transformation of the $\pi$-conjugated carbazole unit and (ii) an evolution of the $\mathrm{CN}$ groups from an extensive conjugation to a nearly isolated (non-conjugated) scenario. More details about the single crystal structure of tetramer 2 are presented in Supporting Information (the corresponding CIF file and Tables S2- S4).

The ${ }^{1} \mathrm{H}$ NMR spectrum of a freshly prepared $\mathrm{CDCl}_{3}$ solution of tetramer $\mathbf{2}$ (dissolved as a white powder) showed signals of only one carbazole unit (Figure S3), being consistent with the result of the single-crystal X-ray diffraction (XRD) analysis. The XRD pattern of the white powder obtained by removing the dichloromethane solvent from the purple solution of $\mathbf{1}$ under vacuum matches with the one simulated from the single-crystal data (Supporting Information, Figure S6). Combining the information from the ${ }^{1} \mathrm{H}$ NMR spectrum and XRD powderdiffraction patterns, we conclude that cyclophane tetramer $\mathbf{2}$ is the only component of the white powder.
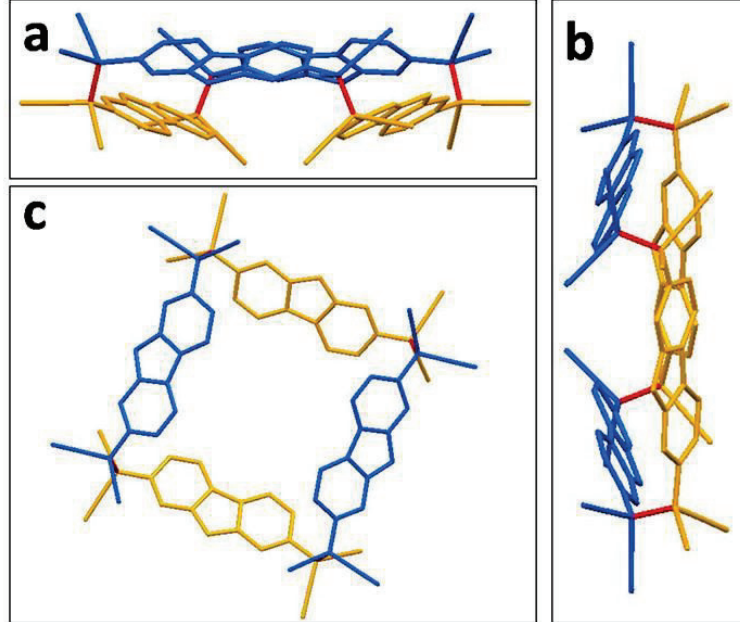

Figure 2. Single crystal structure of tetramer $\mathbf{2}$ (with the alkyl substituents and hydrogen atoms omitted for clarity) viewed along the $a$-axis (a), the $b$-axis (b) and the $c$-axis (c).

\section{DFT calculations}

The formation of tetramer 2 , and the broadened ${ }^{1} \mathrm{H}$ NMR spectra of compound 1 strongly support a strong contribution of the openshell biradical resonance form (i.e., 1-OS in Scheme 1) to the ground state of compound 1 . To further understand the ground state electronic structure of $\mathbf{1}$, density functional theory (DFT) calculations were performed (see Supporting Information for details). The calculated data indicate that $\mathbf{1}$ is an open-shell singlet biradical in the ground state with a small energy differenceof ca. $2 \mathrm{kcal} \mathrm{mol}^{-1}$ between the open-shell and closedshell states and a singlet - triplet energy gap of ca. $5 \mathrm{kcal} \mathrm{mol}^{-1}$ (Supporting Information, Table S1). The moderate singlet-triplet energy gap indicates that, at room temperature, the representation of the triplet state of biradicaloid 1 in solution is low, being consistent with the ${ }^{1} \mathrm{H}$ NMR result. The calculated data reveal that: (i) the singly occupied molecular orbitals (SOMO) and the spin density in open-shell singlet biradical $\mathbf{1}$ are fully delocalized along the whole $\pi$-conjugated backbone (Supporting Information, Figure S7), and (ii) while the closed-shell form has a marked quinoidal pattern, the open-shell singlet biradical form shows certain degree of aromatization of the carbazole unit (Supporting Information, Figures S7-S8). Thus, aromatization acts as a driving force favoring the biradicaloid character of 1 in its ground state.

\section{Electronic absorption spectra}

The UV-vis absorption spectrum of 1 prepared in situ by oxidation of 3 with DDQ, shows strong absorption between 400-650 nm, with several features, which is typically observed in quinoidal compounds (Supporting Information, Figure S9). ${ }^{[17-18]}$ In addition, a broad low-intensity absorption with two spectral features is observed between 650-850 $\mathrm{nm}$. 
1

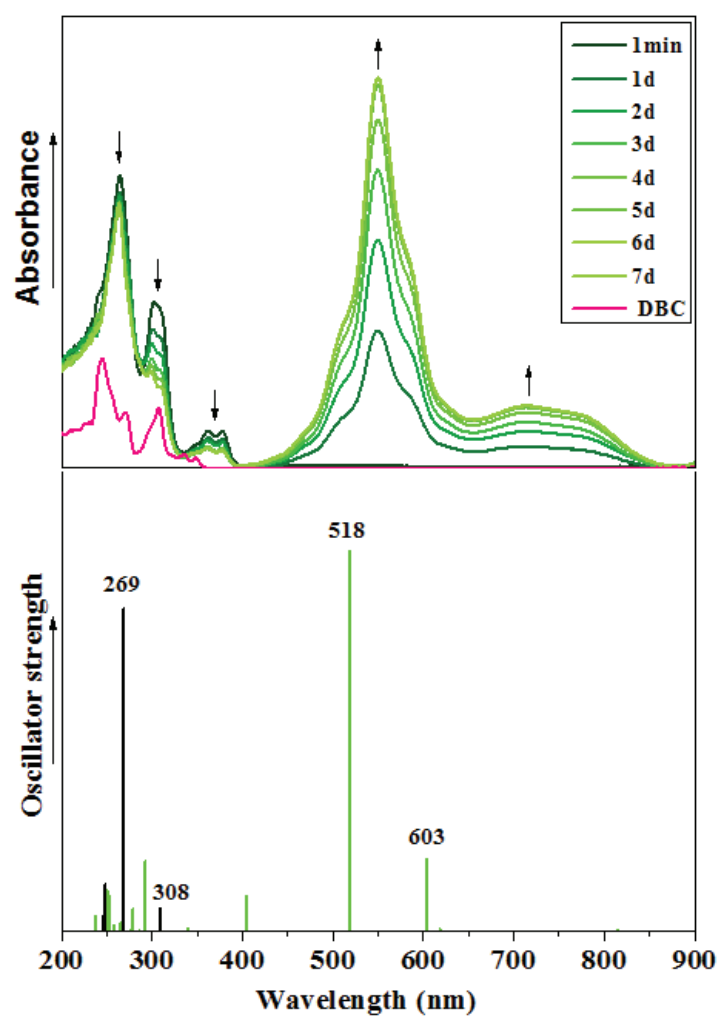

Figure 3. (a) Changes in the UV-vis absorption accompanying the transformation of a freshly prepared solution of $10^{-5} \mathrm{M}$ tetramer 2 $(\downarrow)$ in $\mathrm{CHCl}_{3}$ to biradicaloid $\mathbf{1}(\uparrow)$ at room temperature as a function of time. The spectrum of the precursor 2,7-dibromocarbazole (DBC) in $\mathrm{CHCl}_{3}$ (purple spectrum) is shown for comparison. (b) TD-DFT calculated vertical transition energies for the open-shell singlet biradicaloid $\mathbf{1}$ (green vertical lines) and tetramer $\mathbf{2}$ (black vertical lines) at the M06-2X/6-31G** level.

Its origin very likely lies in admixing the doubly excited electronic configuration (HOMO, HOMO $\rightarrow$ LUMO, LUMO) into the ground state; ${ }^{[23]}$ this feature is characteristic for open-shell polycyclic aromatic hydrocarbons with unpaired electrons, ${ }^{[4]}$ further pointing to a biradicaloid character of compound 1 . When the white powder of pure tetramer $\mathbf{2}$ was dissolved in chloroform, the colorless solution turned gradually purple (as derived from results of Figure 3a). Importantly, the purple solution showed the same absorption spectrum as that of compound 1 prepared according to Scheme 1 , suggesting a gradual thermal transformation of tetramer 2 to monomer $\mathbf{1}$ in this solvent (Supporting Information, Figure S9). The purple solution could be stored at ambient conditions for 6 months with no color decay, demonstrating the stability of $\mathbf{1}$ in the chloroform solution. Time-dependent DFT (TD-DFT) calculations firmly support the spectral evolution observed in the experiment which accompanies the transformation of $\sigma$-bonded tetramer 2 to isolated 1 (see Figure $3 \mathrm{~b}$ and Figure S10-S11). That is, an intense electronic transition is predicted for tetramer 2 at $269 \mathrm{~nm}$, which is associated with a direct optical population of degenerate excited states $S_{0} \rightarrow S_{6} / S_{0} \rightarrow S_{7}$ involving mainly orbitals with $C-C$ bonds having $\sigma$-bonding and $\sigma$-antibonding characters
(Supporting information, Figures S10-S12). The corresponding photochemical reactivity in the $\left(\sigma \sigma^{*}\right)$ excited states is described below. Two intense electronic transitions at 518 and $603 \mathrm{~nm}$ have been calculated for isolated biradicaloid 1 and shown to associate with $\left(\pi \pi^{*}\right)$ excited states $S_{0} \rightarrow S_{3}$ and $S_{0} \rightarrow S_{4}$, respectively (Supporting Information, Figures S11-S13).
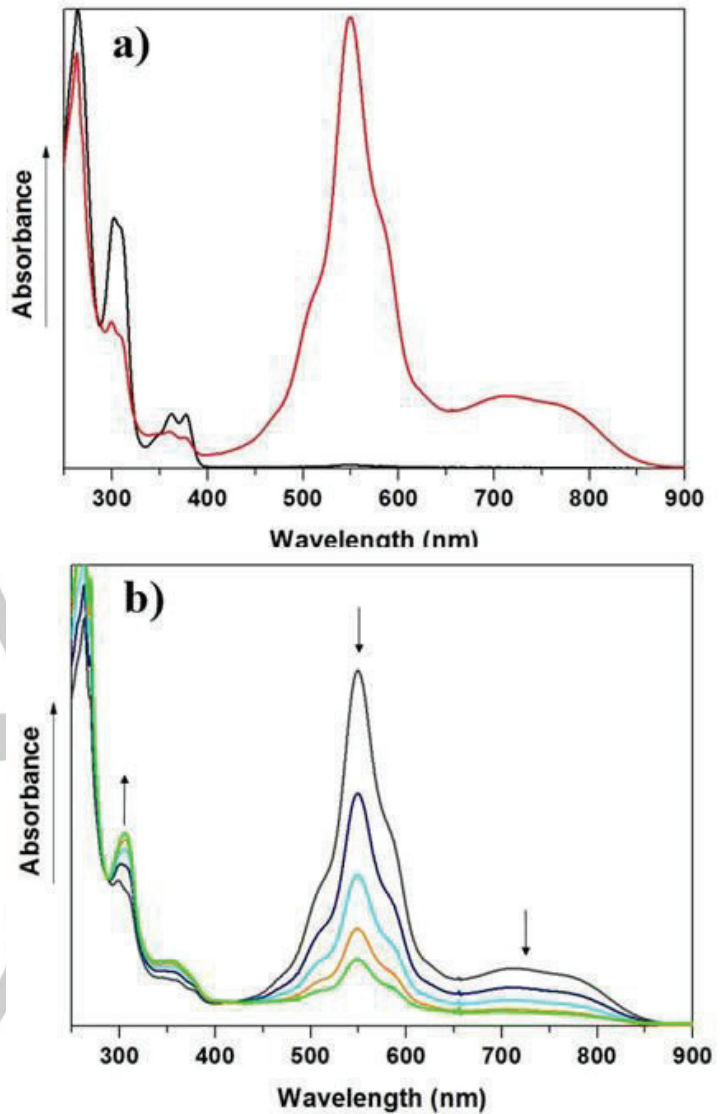

Figure 4. (a) Electronic absorption spectra of $10^{-5} \mathrm{M}$ tetramer 2 in a freshly prepared chloroform solution (black curve) and photoproduct 1 (orange/brown curve) generated rapidly at room temperature upon pulsed-laser UV irradiation $(355 \mathrm{~nm}, 800$ pulses). (b) Partial photochemical recovery of $\mathbf{2}$ in chloroform upon green pulsed-laser irradiation (532 nm) into the dominant $\pi \pi^{*}$ absorption band of 1: dark grey (initial solution), blue navy (800 pulses), cyan (1600 pulses), red (2400 pulses) and green (3200 pulses).

Interestingly, when the purple solution of monomer 1 in chloroform was slowly cooled from $293 \mathrm{~K}$ down to $210 \mathrm{~K}$, the color faded gradually, and the typical UV absorption of tetramer 2 at 250-400 nm increased at the expense of the absorption at $450 \mathrm{~nm}$ and $850 \mathrm{~nm}$ (Supporting information, Figure S14). The purple color recovered quickly when the solution was warmed up to room temperature (Supporting information, Figure S15), thus revealing the reversible thermo-chromic properties of these materials.

Notably, monomer biradicaloid 1 and tetramer $\mathbf{2}$ also display distinct photochromic behavior. The freshly prepared colorless solution of tetramer $\mathbf{2}$ in chloroform turns purple immediately on 
irradiation with UV light $\left(\lambda_{\mathrm{exc}}=355 \mathrm{~nm}\right.$ ), producing biradicaloid 1 (Figure 4a), and returns to its original colorless state by green laser $\left(\lambda_{\text {exc }}=532 \mathrm{~nm}\right)$ irradiation, which leads to a partial recovery of 2 (Figure 4b). Recall that the latter reverse process is not observed thermally in chloroform at ambient temperature. The mechanism of the conversion $\mathbf{2} \rightarrow \mathbf{1}$ upon the UV irradiation can be ascribed to a $\mathrm{C}-\mathrm{C}$ bond cleavage when optically populating the $\left(\sigma \sigma^{*}\right)$ excited states of the tetramer (see above); note that the HOMOs involved in the optically populated excited state of tetramer $\mathbf{2}$ are $\sigma$-bonding with respect to the single bond, $(\mathrm{CN})_{2} \mathrm{C}$ $\mathrm{C}(\mathrm{CN})_{2}$, whereas the LUMOs display a corresponding $\sigma$ antibonding character (Supporting Information, see Figure S12 for the topologies of the relevant molecular orbitals of tetramer 2 ). On the other hand, the reverse conversion $\mathbf{1} \rightarrow \mathbf{2}$ with the green light irradiation is likely associated with an increased biradical character acquired by monomer 1 when optically populating the lowest $\pi \pi^{*}$ (HOMO $\rightarrow$ LUMO and HOMO-1 $\rightarrow$ LUMO) excited states (Supporting Information, Figure S13).

Note that DFT calculations reveal that the electron density on the bridgehead $\mathrm{C}\left(\mathrm{sp}^{3}\right)$ atoms connected to the $\mathrm{CN}$ groups increases significantly on the LUMO occupation, which might favor the formation of the $\mathrm{C}-\mathrm{C} \sigma$-bonds between the excited biradicaloid monomers.

\section{EPR and Raman (resonance and FT) spectroscopies}

At room temperature, X-band EPR spectra of the purple solution in $\mathrm{CH}_{2} \mathrm{Cl}_{2}$ containing monomer $\mathbf{1}$ and tetramer $\mathbf{2}$ in a thermal equilibrium were silent. The only recorded signal was a well resolved quadruplet of the thianthrenium radical cation added as an internal reference. ${ }^{[24-25]}$ Also, the solution of monomer 1 in $\mathrm{CH}_{2} \mathrm{Cl}_{2}$ prepared by in situ oxidation of 3 with $\mathrm{DDQ}$ (Scheme 1) only afforded the strong EPR signal of free DDSQ.[26] The silent EPR responses of 1 support the singlet biradicaloid nature of its ground state ${ }^{[1,4]}$ in agreement with the moderate singlet-triplet energy gap obtained from DFT calculations.

Raman spectroscopy has been demonstrated to be a sensitive technique to probe the biradicaloid character of quinoidal type compounds. [9] Figure $5 \mathrm{a}$ displays the resonance Raman spectrum (RRS) of monomer 1 formed by in situ oxidation of 3 with $\mathrm{DDQ}$ in dichloromethane at room temperature, and the FTRaman spectrum of tetramer 2 as a white powder. Both Raman spectra display intense bands in the $1600-1100 \mathrm{~cm}^{-1}$ region together with an intense $\mathrm{v}(\mathrm{CN})$ band at around $2200 \mathrm{~cm}^{-1}$; a wavenumber decrease is typically observed for monomer 1 compared to tetramer $\mathbf{2}$. The most intense band of tetramer 2 appears at $1634 \mathrm{~cm}^{-1}$, belonging to a $\mathrm{v}(\mathrm{CC})$ mode of the carbazole rings. This mode appears at $1627 \mathrm{~cm}^{-1}$ for an unsubstituted carbazole (Supporting Information, Figure S16), but at $1598 \mathrm{~cm}^{-}$ ${ }^{1}$ for monomer 1 , suggesting partial aromatization of the carbazole unit in line with the DFT calculations (Supporting Information, Figure S9). In the same context, the CN stretching frequency, $\mathrm{v}(\mathrm{CN})$, of tetramer $\mathbf{2}$, which is close to that of non-conjugated nitriles (e.g., around $2270 \mathrm{~cm}^{-1}$ in malononitrile), shifts from 2253 $\mathrm{cm}^{-1}$ in tetramer 2 to $2210 \mathrm{~cm}^{-1}$ in monomer 1 . The smaller value of $\mathrm{v}(\mathrm{CN})$ confirms that the $\mathrm{CN}$ groups of 1 are conjugated with the central carbazole ring. Again, the spectral trend is well reproduced by our DFT calculations for isolated biradicaloid monomer 1 and $\boldsymbol{\sigma}$-bonded tetramer $\mathbf{2}$ (Figure $5 \mathrm{~b}$ ), and in good agreement with the calculated bond length changes in monomer 1 and $\sigma$-bonded tetramer 2 (Supporting Information, Figure S8). The resonance Raman spectrum of the purple species forming slowly from 2 in chloroform solution at room temperature over a period of $5 \mathrm{~h}$ (Supporting information, Figure S17) is the same as that of monomer $\mathbf{1}$, further proving the conversion of tetramer 2 to monomer 1 in solution.

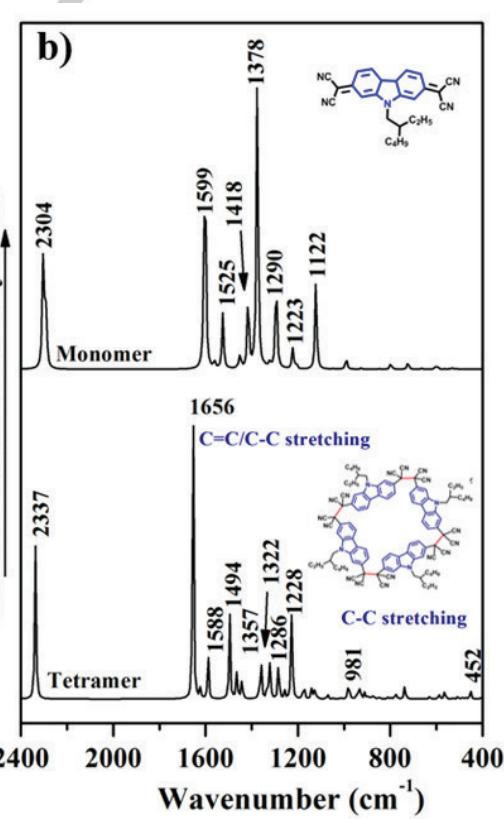

Figure 5. (a) FT-Raman spectrum of solid tetramer $2\left(\lambda_{\text {exc }}=1064 \mathrm{~nm}\right.$, bottom) and RRS spectrum ( $\lambda_{\text {exc }}=532 \mathrm{~nm}$, top) of 1 in dichloromethane solution formed by in situ oxidation of 3 with DDQ. (b) Theoretical Raman spectra calculated (M06$\left.2 X / 6-31 G^{* *}\right)$ for isolated biradical 1 and tetramer 2. 
Interconversion of biradicaloid $\mathbf{1}$ and tetramer $\mathbf{2}$ in solid state

In addition to the thermal equilibrium between 2 and 1 in solution, the transformation of tetramer $\mathbf{2}$ to monomer biradicaloid 1 can also readily be achieved by heating and/or applying external pressure in the solid state, which was monitored by IR and Raman spectroscopies and XRD. Figure 6a shows the IR spectrum (in ATR configuration) of solid tetramer $\mathbf{2}$ as a white powder and after grinding used to prepare a $\mathrm{KBr}$ pellet. The $v(\mathrm{CN})$ band of the white powder appears at $2253 \mathrm{~cm}^{-1}$, which is close to that found in nonconjugated nitriles. Grinding the white solid powder results in a purple color and a new $v(\mathrm{CN})$ band rising around $2206 \mathrm{~cm}^{-1}$, which is assigned to biradicaloid 1 and confirms the huge effect of strain/pressure on the transformation. Interestingly, upon temperature cycling between room and $523 \mathrm{~K}$ of the $\mathrm{KBr}$ pellet of tetramer 2 the $2253 \mathrm{~cm}^{-1}$ band almost completely disappeared, while the $2206 \mathrm{~cm}^{-1}$ band increased its intensity. The $v(\mathrm{CN})$ wavenumber downshift indicates that the $\mathrm{CN}$ groups evolve from a nearly isolated scenario in $\sigma$-bonded tetramer $\mathbf{2}$ to fully conjugated one in isolated monomer biradicaloid 1.

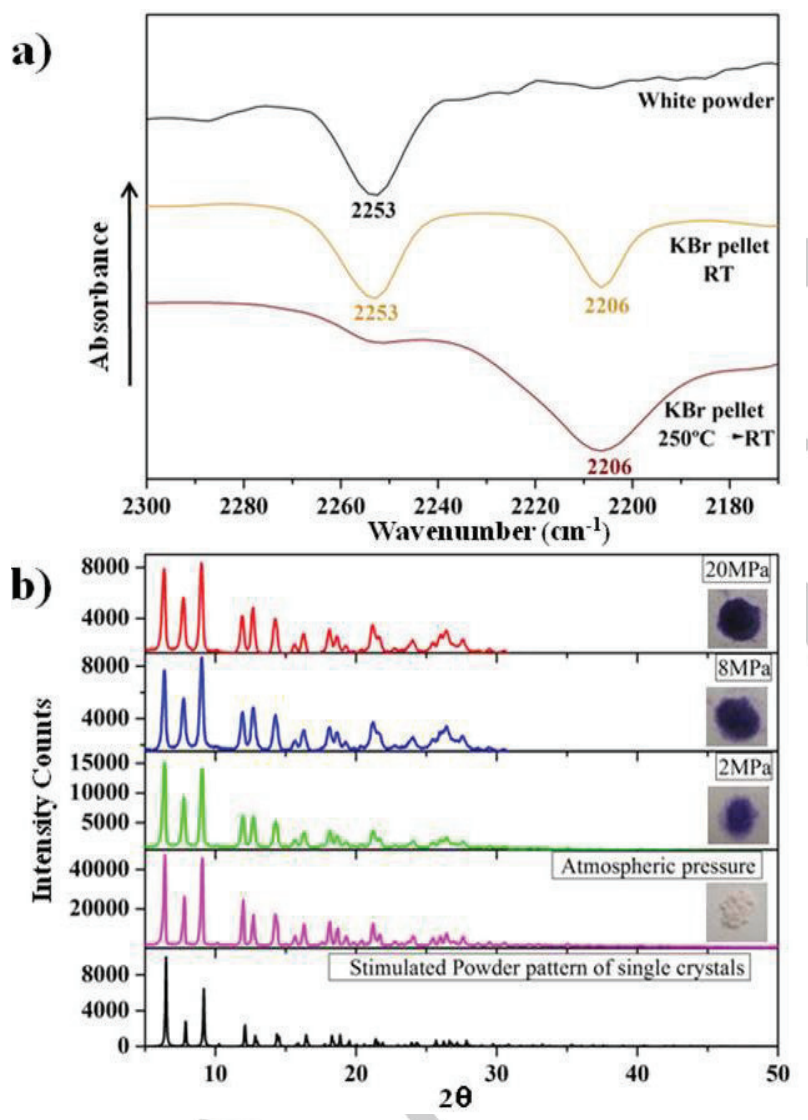

Figure 6. a) IR spectrum of tetramer 2 as a white powder at room temperature (top), a purple $\mathrm{KBr}$ pellet containing a mixture of tetramer $\mathbf{2}$ and biradicaloid $\mathbf{1}$ obtained after grinding $\mathbf{2}$ at room temperature (middle), and the $\mathrm{KBr}$ pellet with dominant purple biradicaloid 1 after heating to $523 \mathrm{~K}$ and subsequent cooling down to room temperature (bottom). b) The changes in color and XRD patterns for tetramer 2 at pressures up to $20 \mathrm{MPa}$.
Color changes observed in tetramer 2 resulting from the application of mild pressures (up to $20 \mathrm{MPa}$ ) are shown in the inserted images of Figure $6 \mathrm{~b}$. The powder XRD results plotted along in the same figure clearly reveal the decreased intensity of the diffraction peaks due to diminished $\mathbf{2}$ at the higher pressures, while no clear new diffraction peaks appear. Combining the UVVis, IR, RRS and XRD data, the color changes are beyond any doubt due to the transformation of tetramer 2 to biradicaloid 1 . Since the conversion of $\mathbf{2}$ to monomer $\mathbf{1}$ could also be modulated by applying small external pressures, we performed additional Raman spectroscopy experiments to pressures in the GPa range (Figure 7) seeking for further transformations (Supporting Information, Figures S18-S21). We observe that at compressions by ca. $0.7 \mathrm{GPa}$ the tetramer is broken and a monomeric configuration is favored, as revealed by the changes in the Raman spectrum of the sample: (i) vanishing of the bands at 2253 and $1634 \mathrm{~cm}^{-1}$ belonging to the $\mathrm{v}(\mathrm{CN})$ and $\mathrm{v}(\mathrm{CC})$ modes in the carbazole rings, respectively, and at $416 \mathrm{~cm}^{-1}$ assigned to $\sigma$-bond stretching, $\mathrm{v}(\mathrm{CC})$, and (ii) the prevalence of the bands of the quinoid carbazole at 2210 and $1600 \mathrm{~cm}^{-1}$ due to the $\mathrm{v}(\mathrm{CN})$ and $\mathrm{v}(\mathrm{CC})$ modes, respectively. Stronger compression induces molecular rearrangements with the potential formation of $\sigma$ oligomers. This suggestion is supported by the increase in intensity of the $\mathrm{v}(\mathrm{CC})$ band at $419 \mathrm{~cm}^{-1}$ assigned to $\sigma$-bond stretching, and the shift of the $\mathrm{v}(\mathrm{CC})$ mode of the carbazole rings to $1618 \mathrm{~cm}^{-1}$, which suggest oligomeric structures with less aromatic cores than in an isolated tetramer.

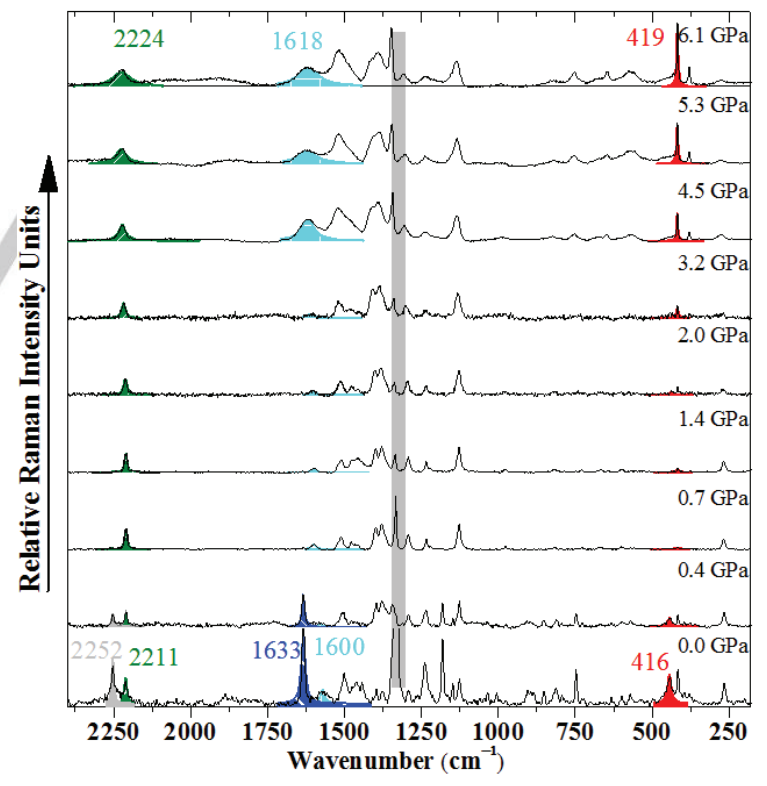

Figure 7. Raman spectra of tetramer 2 at selected pressures, measured at $532 \mathrm{~nm}$ excitation. Marked peaks correspond to: the $\mathrm{v}(\mathrm{CC})$ mode of a $\sigma$ bond, red colored area; the $\mathrm{v}(\mathrm{CC})$ mode of the tetramer carbazole rings, dark blue area; the $\mathrm{v}(\mathrm{CC})$ mode assigned to $\sigma$-bonded oligomers, cyan area; the $\mathrm{v}(\mathrm{CN})$ modes, grey and green area. The shadowed region marks the band of the diamond chip used as pressure marker. 


\section{Conclusions}

Here we investigate a novel three-ring $\pi$-conjugated singlet biradicaloid compound $\mathbf{1}$ which forms a macrocycle cyclophanetype tetramer 2 . Single crystal $\mathrm{X}$-ray diffraction of $\mathbf{2}$ revealed the formation of four long $(\mathrm{CN})_{2} \mathrm{C}-\mathrm{C}(\mathrm{CN})_{2}$ bonds (ca. $1.631 \AA$ ) by sharing the unpaired electrons of four molecules of biradicaloid 1 . Importantly, this work demonstrates for the first time reversible photoswitching of $\sigma$-aggregated compounds formed from a stable quinoidal precursor. In fact, a dynamic and reversible cyclic transformation between monomer $\mathbf{1}$ and tetramer $\mathbf{2}$ is observed upon external stimuli (light absorption temperature and moderate pressures), both in solution and solid state, showing a strong violet to pale chromism. Interestingly, the reversible interconversion between $\mathbf{1}$ and $\mathbf{2}$ is hindered when applying high pressure $(\mathrm{GPa})$, opening the possibility for the formation of new $\sigma$-oligomers. Thus, these soft materials can be envisaged as promising functional materials for practical applications in sensors, molecular (photochromic) switches, optoelectronics and dynamic covalent chemistry.

\section{ASSOCIATED CONTENT}

Supporting Information. Experimental section, absorption spectra, Raman spectra, DFT calculations and X-Ray details. Please note that the CIF file of tetramer 2 has been deposited at the Cambridge Crystallographic Data Center (CCDC) with a CCDC number of 1484203 . The supporting information material is available free of charge via the Internet at http://pubs.acs.org.

\section{AUTHOR INFORMATION}

\section{Corresponding Author}

* Ihx@mail.sioc.ac.cn

*carmenrd@uma.es

*f.hartl@reading.ac.uk

\section{Acknowledgment}

This work was supported by the National Natural Sciences Foundation of China (Projects 21190031, 51273212) and the "Strategic Priority Research Program" of the Chinese Academy of Sciences (Project XDB12010100). F. H. acknowledges support from the University of Reading (Projects D14-015 and EPSRC DTP). The work at the University of Malaga was supported by MINECO (project reference CTQ2015-66897-P) and Junta de Andalucia (P09-FQM-4708). The work at Complutense University was supported by MINECO under project CTQ2015-67755-C0201. We thank also Prof. Mercedes Taravillo for her support in the pressure dependent experiments. R.P.O. thanks the MICINN for a "Ramon y Cajal" Research contract and S. G.-V. thanks the University of Málaga for his grant.

\section{References}

1. M. Abe, Chem. Rev. 2013, 113, 7011-7088.

2. Z. Zeng, X. Shi, C. Chi, J.T. L. Navarrete, J. Casado, J. Wu, Chem. Soc. Rev. 2015, 44, 6578-6596.
3. J. Lee, P. Jadhav, P. D. Reusswig, S. R. Yost, N. J. Thompson, D. N. Congereve, E. Hontz, T. V. Voorhis, M. A. Baldo, Acc. Chem. Res. 2013, 46, 1300-1311.

4. Z. Zeng, Y. M. Sung, N. Bao, D. Tan, R. Lee, J. L. Zafra, B. S. Lee, M. Ishida, J. Ding, J. T. L. Navarret, Y. Li, W. Zeng, D. Kim, K. Huang, R. D. Webster, J. Casado, J. Wu J. Am. Chem. Soc. 2012, 134, 14513-14525

5. Y. Li, W. Heng, B. S. Lee, N. Aratani, J. L. Zafra, N. Bao, R. Lee, Y. M. Sung, Z. Sun, K. Huang, R. D. Webster, J. T. L. Navarrete, D. Kim, A. Osuka, J. Casado, J. Ding, J. Wu, J. Am. Chem. Soc. 2012, 134, 14913-14922.

6. X. Zhu, H. Tsuji, K. Nakabayashi, S. Ohkoshi, E. Nakamura, J. Am. Chem. Soc.2011, 133, 16342-16345.

7. Z. Sun, K. Huang, J. Wu, J. Am. Chem. Soc. 2011, 133, 1189611899.

8. R. P. Ortiz, J. Casado, V. Hernandez, J. T. L. Navarret, E. Orti, P. M. Viruela, B. Milian, S. Hotta, G. Zotti, S. Zecchin, B. Vercelli, Adv. Funct. Mater. 2006, 16, 531-536.

9. E. V. Canesi, D. Fazzi, L. Colella, C. Bertarelli, C. Castiglioni, J. Am. Chem. Soc.2012, 134, 19070-19083.

10. A. Shimizu, Y. Tobe, Angew. Chem. Int. Ed.2011, 50, 6906-6910.

11. G. E. Tudebusch, J. L. Zafra, K. Jorner, K. Fukuda, J. L. Marshall, I. Arrechea-Marcos, G. L. Espejo, R. P. Ortiz, C. J. Gomez-Garcia, L. N. Zakharov, M. Nakano, H. Ottosson, J. Casado, M. M. Haley, Nature Chemistry, 2016, 8, 753-759.

12. Y. Jin, Q. Wang, P. Taynton, W. Zhang, Acc. Chem. Res., 2014, 47, 1575-1586.

13. Y. Jin, C. Yu, R. J. Denman, W. Zhang, Chem. Soc. Rev., 2013, 42, 6634-6654.

14. T. Kabashi, D. Sakamaki, S. Seki,_Angew. Chem. Int. Ed. 2016, 55, 8634-8638

15. Zafra, J. L.; Q. L.; Yanai, N.; Mori, Takamichi, Miriam, M. N. ; Alvarez, M. P. ; Navarret, J. T. L.; Gomez-Garcia, C. J.; Kertesz, M.; Takimiya, K.; Casado, J. Angew. Chem. Int. Ed. 2016, $55,14563-14568$

16. Q. Wu, R. Li, W. Hong, H. Li, X. Gao, D. Zhu, Chem. Mater. 2011, 23, 3138-3140.

17. J. Li, X. Qiao, Y. Xiong, H. Li, Chem. Mater. 2014, 26, 5782 5788.

18. Q. Wu, S. Ren, M. Wang, X. Qiao, H. Li, X. Gao, X. Yang, D. Zhu, Adv. Funct. Mater. 2013, 23, 2277-2284.

19. C. Zhang, Y. Zang, E. Gann, C. R. McNeill, X. Zhu, C. Di, D. Zhu, J.Am. Chem. Soc. 2014, 136, 16176-16184.

20. T. Takahashi, K. Matsuoka, K. Takimiya, T. Otsubo, Y. Sao, J. Am. Chem. Soc. 2005, 127, 8928-8929.

21. M. Frasconi, I. R. Fernando, Y. Wu, Z. Liu, W. Liu, S. M. Dyar, G. Barin, M. R. Wasielewski, W. A. Goddard, J. F. Stoddart,J. Am. Chem. Soc. 2015, 137, 11057-11068.

22. E. J. Dale, N. A. Vermeulen, M. Juricek, J. C. Barnes, R. M. Young, M. R. Wasielewski, J. F. Stoddart,Acc. Chem. Res. 2016, 49, 262-273.

23. P. Rapta, L. Kress, P. Hapiot, L. Dunsch, PCCP2002, 4, 41814185.

24. H. J. Shine, C. F. Dais, R. J. Small, J. Org. Chem. 1964, 29, 2125.

25. S. D. Motta, F. Negri, D. Fazzi, C. Castiglioni, E. V. Canesi, J. Phys. Chem. Lett. 2010, 1, 3334-3339.

26. D. Gordon; M. J. Hove, J. Chem. Phys. 1973, 59, 3419-3420. 
WILEY-VCH FULL PAPER

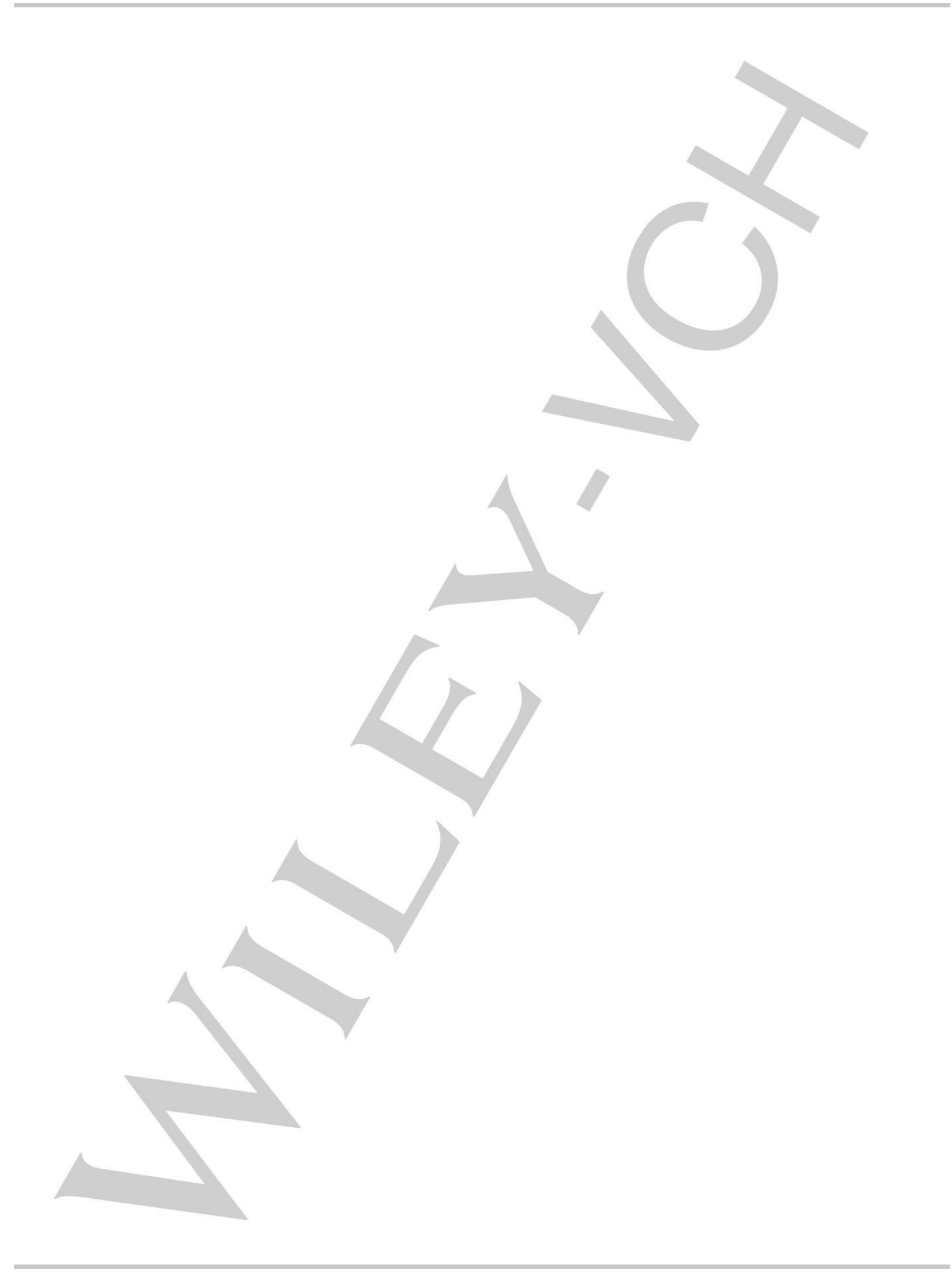


WILEY-VCH FULL PAPER

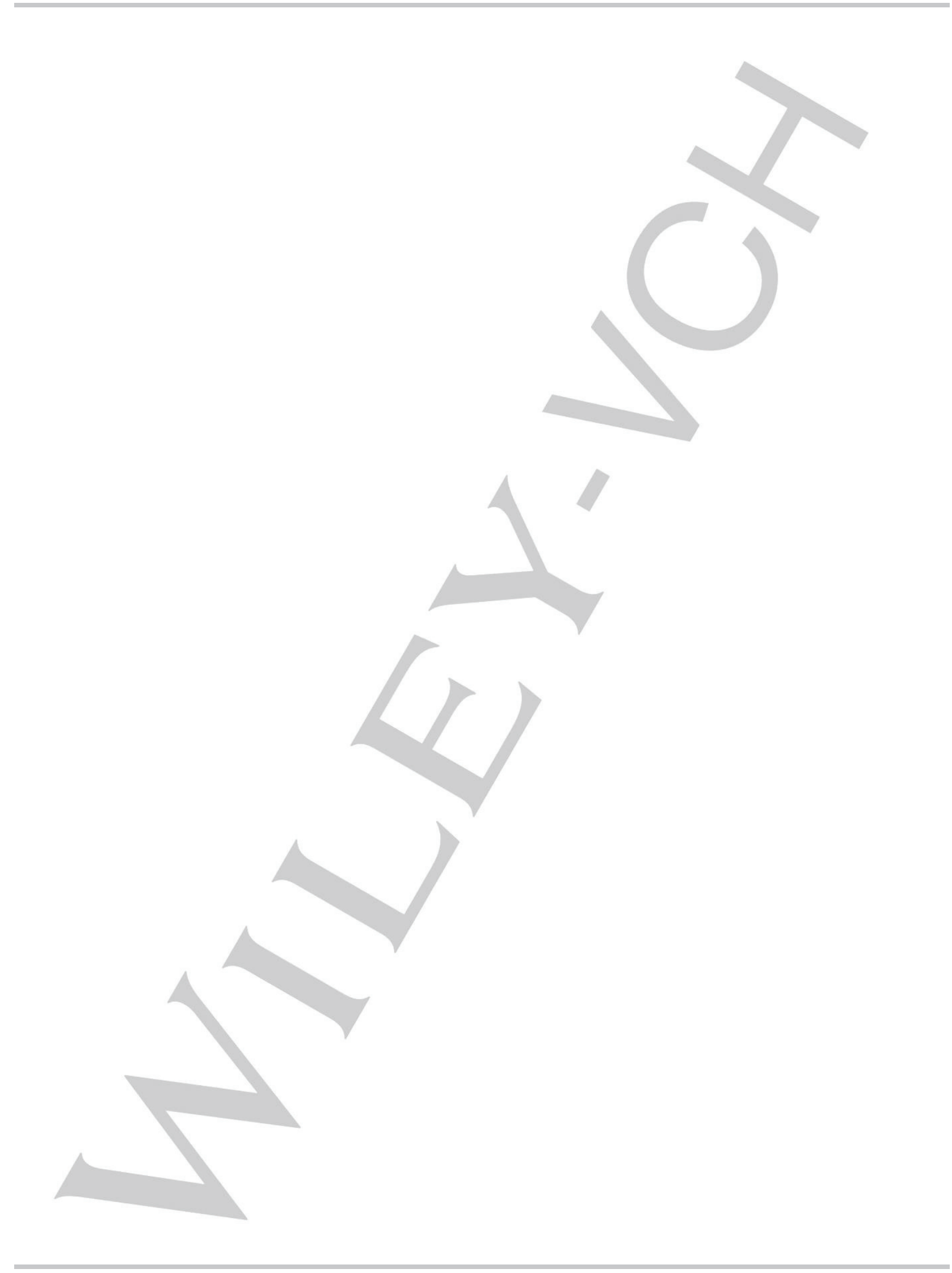

